\title{
A novel endoplasmic stress mediator, Kelch domain containing 7B (KLHDC7B), increased Harakiri (HRK) in the SubAB-induced apoptosis signaling pathway
}

\author{
Kinnosuke Yahiro $\mathbb{D}^{1 凶}$, Kohei Ogura ${ }^{2}$, Hiroyasu Tsutsuki ${ }^{3}{ }^{3}$, Sunao lyoda ${ }^{4}$, Makoto Ohnishi ${ }^{4}$ and Joel Moss ${ }^{5}$
}

(c) The Author(s) 2021

Locus for Enterocyte Effacement (LEE)-positive Shiga-toxigenic Escherichia coli (STEC) contributes to many global foodborne diseases, with infection characterized by severe gastrointestinal symptoms, including bloody diarrhea. The incidence of LEEnegative STEC-mediated disease is also increasing globally. Subtilase cytotoxin (SubAB) is released by some LEE-negative STEC strains. It cleaves $\mathrm{BiP}$, which is a chaperone protein located in the endoplasmic reticulum (ER), thereby causing apoptosis induced by ER stress. To date, the apoptotic signaling pathway mediated by SubAB has not been identified. In the current study, RNA-seq analysis showed that SubAB significantly induced the expression of Kelch domain containing 7B (KLHDC7B). We explored the role of KLHDC7B in the SubAB-induced apoptotic pathway. SubAB-induced KLHDC7B mRNA expression was increased after $12 \mathrm{~h}$ of incubation of toxin with HeLa cells. KLHDC7B expression was downregulated by knockdown of PKR-like endoplasmic reticulum kinase (PERK), CEBP homologous protein (CHOP), activating transcription factor 4 (ATF4), and CEBP $\beta$ (CEBPB). KLHDC7B knockdown suppressed SubAB-stimulated CHOP expression, poly(ADP-ribose) polymerase (PARP) cleavage, and cytotoxicity. The overexpressed KLHDC7B was localized to the nucleus and cytosolic fractions. Next, we used RNA-seq to analyze the effect of KLHDC7B knockdown on apoptosis induced by SubAB, and found that the gene encoding for the pro-apoptotic Bcl-2 family protein, Harakiri (HRK), was upregulated in SubAB-treated control cells. However, this effect was not observed in SubAB-treated KLHDC7Bknockdown cells. Therefore, we identified the pathway through which SubAB-induced KLHDC7B regulates HRK expression, which is essential for apoptosis in toxin-mediated ER stress.

Cell Death Discovery (2021)7:360 ; https://doi.org/10.1038/s41420-021-00753-0

\section{INTRODUCTION}

Serotype 0157:H7 is the most common strain of Locus for Enterocyte Effacement (LEE)-positive, Shiga-toxigenic Escherichia coli (STEC). This serotype causes many food-borne diseases, which manifest as bloody diarrhea, hemorrhagic colitis, and hemolyticuremic syndrome (HUS) [1]. Shiga toxin (Stx) 1 and/or 2 produced by most LEE-positive STEC are important virulence factors that trigger severe gastrointestinal diseases and HUS [2]. LEE-negative STEC strains may also cause infections, and the virulence of some of these strains results from the production of the cytotoxins, Stx2 and subtilase cytotoxin (SubAB) [3].

SubAB and Stx are cytotoxins that belong to the $A B_{5}$ family [3], and are comprised of a catalytically active $A$ subunit and a $B$ subunit pentamer, which contains the receptor-binding domain [3]. SubAB, produced by LEE-negative STEC, recognizes sialic acid-modified glycoproteins on the human cell surface as receptors [4-6]. Binding to the receptor internalizes the toxin by endocytosis [7] and macropinocytic-like pathways [8]. SubAB is translocated from the Golgi to endoplasmic reticulum (ER) via pathways that involve the coatomer protein complex subunit Beta, sorting nexin, component oligomeric Golgi complex, Ras-related proteins [9], jumping translocation breakpoint protein, KDEL endoplasmic reticulum protein retention receptor 2 [6], and protein disulfide isomerase [10]. SubAB cleaves the chaperone protein BiP/Grp78 $\left(\right.$ Leu $^{416 / 417}$ ) at a specific site [3], thereby activating proteins that detect ER stress, e.g., PERK, inositol-requiring enzyme (IRE1), and activating transcription factor 6 (ATF6) [11, 12]. These events are followed by caspase activation via mitochondrial cytochrome $c$ release into the cytosol [13]. Furthermore, stress signaling inhibits protein synthesis [14], causes cell cycle arrest [14], decreases iNOS synthesis [15], promotes the formation of stress granules [16], and induces the production of a novel, nonsecretory form of lipocalin 2 [17]. Additionally, when SubAB is administered intraperitoneally into the mice, severe fatal intestinal hemorrhage occur [18].

Kelch domain containing 7B (KLHDC7B) is a tumor marker with epigenetic differences in breast [19] and laryngeal [20] cancers. The promoter region of KLHDC7B is hyper-methylated in tumors compared to normal tissues [21]. KLHDC7B contains 594 amino acids, with a Kelch domain that, in generally, consists of five to seven repeated motifs [22]. KLHDC7B is a member of the Kelch

\footnotetext{
${ }^{1}$ Department of Microbiology and Infection Control Sciences, Division of Biological Sciences, Kyoto Pharmaceutical University, Kyoto, Japan. ${ }^{2}$ Advanced Health Care Science Research Unit, Institute for Frontier Science Initiative, Kanazawa University, Kumamoto, Kumamoto, Japan. ${ }^{3}$ Department of Microbiology, Graduate School of Medical Sciences, Kumamoto University, Kumamoto, Kumamoto, Japan. ${ }^{4}$ Department of Bacteriology I, National Institute of Infectious Diseases, Tokyo, Japan. ${ }^{5}$ Pulmonary Branch, National Heart, Lung, and Blood Institute, National Institutes of Health, Bethesda, MD, USA. ${ }^{\circledR}$ email: kin816tas@mb.kyoto-phu.ac.jp
} 
A

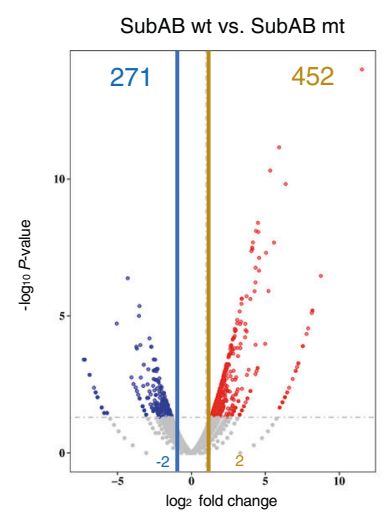

B
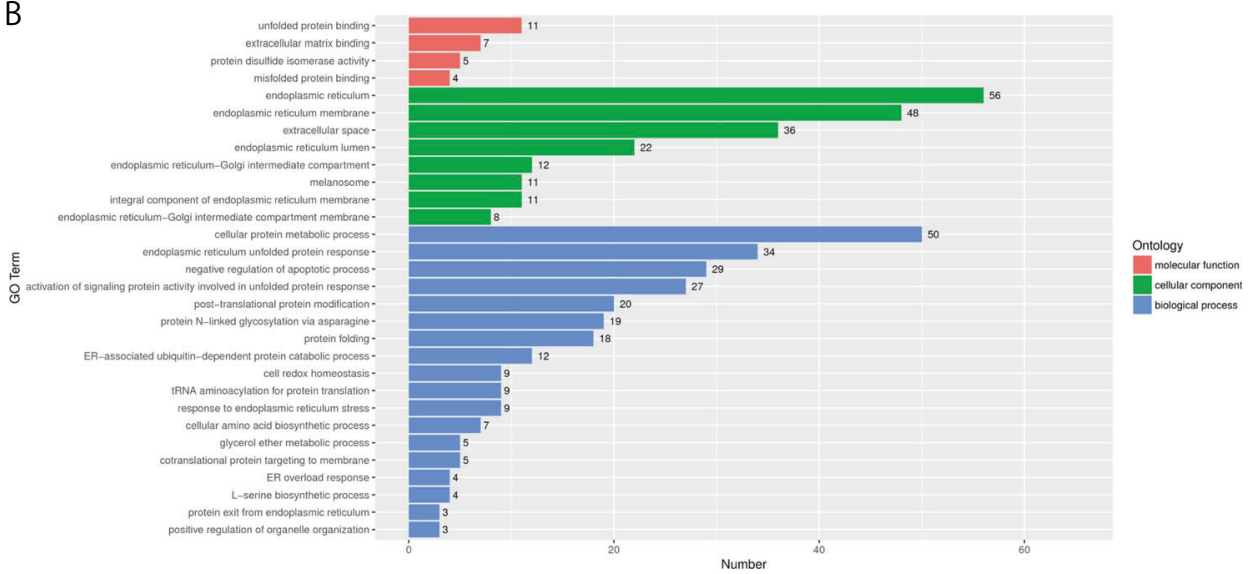

Fig. 1 RNA-seq analysis of SubAB-treated HeLa cells. HeLa cells were incubated with $400 \mathrm{ng} \mathrm{mL}^{-1}$ of SubAB wild-type (wt) or inactivated mutant ( $\mathrm{mt}$ ) for $18 \mathrm{~h}$, followed by RNA extraction. RNA-seq analysis was performed using GENEWIZ. A Volcano plot of differentially expressed genes (DEGs). DEGs were identified using the DESeq2 Bioconductor package, which is based on the negative binomial distribution. Dispersion and logarithmic fold change estimates included data-driven prior distributions; Padj of genes were set at $<0.05$ to identify DEGs. B Gene ontology (GO) term enrichment analysis of DEGs; increased GO terms in biological process, molecular function, and cellular component branches are displayed.

Table 1. The top 10 mRNA enriched by SubAB are listed.

\begin{tabular}{|c|c|c|}
\hline GeneSymbol & Description & Log2 ration \\
\hline KLHDC7B & kelch domain containing 7B & 11.531 \\
\hline ADM2 & adrenomedullin 2 & 5.9341 \\
\hline SLC7A11 & solute carrier family 7 & 5.3272 \\
\hline $\mathrm{CHAC1}$ & cation transport regulator homolog 1 & 6.37 \\
\hline DDIT3 & DNA-damage-inducible transcript 3 & 4.4997 \\
\hline ASNS & $\begin{array}{l}\text { asparagine synthetase (glutamine- } \\
\text { hydrolyzing) }\end{array}$ & 4.3619 \\
\hline GDF15 & growth differentiation factor 15 & 4.524 \\
\hline HSPA5 & heat shock $70 \mathrm{kDa}$ protein 5 & 4.1605 \\
\hline LCN2 & lipocalin 2 & 5.5862 \\
\hline HERPUD1 & $\begin{array}{l}\text { homocysteine-inducible endoplasmic } \\
\text { reticulum stress-inducible ubiquitin- } \\
\text { like domain member } 1\end{array}$ & 4.1037 \\
\hline
\end{tabular}

family; the Kelch proteins play a role in various cellular events (e.g., cytoskeletal arrangement, cell morphology, protein degradation, gene expression) [23]. The cellular function of KLHDC7B is unclear.

Using RNA-seq analysis, we found that KLHDC7B mRNA was significantly increased in SubAB-treated cells $12 \mathrm{~h}$ after incubation.
To determine the function and regulation of KLHDC7B in the SubAB-induced cell death signaling pathway, we analyzed RNAseq data between control and KLHDC7B-knockdown cells with or without SubAB. SubAB-induced KLHDC7B was regulated by the PERK/ATF4/CEBPB/CHOP pathway. KLHDC7B was localized to the cytosolic fraction and nucleus. Harakiri (HRK) belongs to the $\mathrm{BCl}-2$ family and has pro-apoptotic functions. The level of HRK mRNA was significantly enhanced in SubAB-treated control cells, but not KLHDC7B-knockdown cells. Thus, HRK is a critical, pro-apoptotic protein controlled by SubAB-induced KLHDC7B. In this study, we identified the toxin-induced apoptosis pathway involved in the regulation of HRK expression (a critical cell death protein) by SubAB-stimulated KLHDC7B (a novel ER-stress death mediator).

\section{RESULTS}

RNA-seq and differential expression analysis of SubAB-treated and control cells

We used RNA-seq analysis to identify the novel cell death-related protein induced by SubAB in HeLa cells. The differentially expressed genes (DEGs) of SubAB-treated cells showed differences in levels of more than 2 -fold with significant $p$ values $(<0.05)$. The gene expression patterns are depicted in the volcano plot analysis (Fig. 1A), along with the significantly increased (red dots, 452 genes) and decreased (blue dots, 271 genes) genes in SubAB-treated cells.

Gene Ontology (GO) analysis was used to determine the functional and biological processes active in SubAB-treated 
Table 2. List of primers used in this study.

\begin{tabular}{l} 
Primer name \\
FLAG-tagged KLHDC7B forward \\
FLAG-tagged KLHDC7B reverse \\
qPCR KLHDC7B forward \\
qPCR KLHDC7B reverse \\
qPCR CHOP forward \\
qPCR CHOP reverse \\
qPCR GAPDH reverse \\
qPCR GAPDH forward \\
qPCR CEBPB forward \\
qPCR CEBPB reverse \\
qPCR CAPN5 forward \\
qPCR CAPN5 reverse \\
qPCR GAPDH forward \\
qPCR GAPDH reverse \\
qPCR HERPUD1 forward \\
qPCR HERPUD1 reverse \\
qPCR CRELD2 forward \\
qPCR CRELD2 reverse \\
qPCR NUPR1 forward \\
qPCR NUPR1 reverse \\
qPCR GADD45A forward \\
qPCR GADD45A reverse \\
qPCR ATP8B2 forward \\
qPCR ATP8B2 reverse \\
qPCR GADD34 forward \\
qPCR GADD34 reverse \\
qPCR PDIA4 forward \\
qPCR PDIA4 reverse \\
qPCR HNF4A forward \\
qPCR HNF4A reverse \\
qPCR HRK reverward \\
\hline
\end{tabular}

Primer name

FLAG-tagged KLHDC7B reverse

qPCR KLHDC7B forward

qPCR KLHDC7B reverse

QPCR CHOP reverse

qPCR GAPDH reverse

QPCR GAPDH forward

QPCR CEBPB reverse

qPCR CAPN5 forward

GPCR GAPDH forward

qPCR GAPDH reverse

qPCR CRELD2 forward

qPCR CRELD2 reverse

qPCR GADD45A forward

qPCR GADD45A reverse

qPCR ATP8B2 forward

qPCR ATP8B2 reverse

qPCR GADD34 forward

qPCR GADD34 reverse

qPCR PDIA4 forward

qPCR PDIA4 reverse

qPCR HNF4A forward

qPCR HRK forward

qPCR HRK reverse

\section{Sequences}

5'-CAA GCT TGC GGC CGC CAC CATGATCCAGGGCACCTTG-3'

5'-ACCGGATCCGTCGAC GAGTGAGGTCTGCAGCCGGT-3'

5'-GCACAACTACCTGTTTCTGGCG-3'

5'-TGGCTCCAGATGTTGGTCAGAG-3'

5'-GGTATGAGGACCTGCAAGAGGT-3'

5'-CTTGTGACCTCTGCTGGTTCTG-3'

5'-GTCTCCTCTGACTTCAACAGCG-3'

5'-ACCACCCTGTTGCTGTAGCCAA-3'

5'-AGAAGACCGTGGACAAGCACAG-3'

5'-CTCCAGGACCTTGTGCTGCGT-3'

5'-AGTGTGAGGGAGACAAAGTCCG-3'

5'-CATCCTTCAGCACTCGGTGGTT-3'

5'-GTCTCCTCTGACTTCAACAGCG-3'

5'-ACCACCCTGTTGCTGTAGCCAA-3'

5'-CCAATGTCTCAGGGACTTGCTTC-3'

5'-CGATTAGAACCAGCAGGCTCCT-3'

5'-AGAGACTGCGGCGAGTGTGAAG-3'

5'-TAGGAGCCGTTGGCGTTCTTAC-3'

5'-GACTCCAGCCTGGATGAATCTG-3'

5'-CTTCTCTCTTGGTGCGACCTTTC-3'

5'-CTGGAGGAAGTGCTCAGCAAAG-3'

5'-AGAGCCACATCTCTGTCGTCGT-3'

5'-CGGCTATTCCTGCAAGATGCTG-3'

5'-GTCCTGATAGGTGAAGCCGTTG-3'

5'-TCCGACTGCAAAGGCGGCTCA-3'

5'-CAGCCAGGAAATGGACAGTGAC-3'

5'-CCAGCAGGTTTGATGTGAGTGG-3'

5'-GGAGACTTCTCTGACCTTGGCA-3'

5'-GGTGTCCATACGCATCCTTGAC-3'

5'-AGCCGCTTGATCTTCCCTGGAT-3'

5'-AGGTTGGTGAAAACCCTGTG-3'

5'-TTTCTACGATCGCTCCAGGC-3' cells (Fig. 1B). The SubAB-enriched genes were participating in the biological processes of cellular protein metabolism and ER unfolded-protein response. Additionally, for the cellular components, genes involved in the ER or ER membrane were increased.

Table 1 summarizes the top 10 enriched mRNAs from SubABtreated cells. SubAB-upregulated mRNAs (e.g., CHOP [13, 24], HSPA5 [25], CHAC1 [26], LCN2 [17], GDF15 [27], ASNS [28], HERPUD1 [29], ADM2 [30]) were affected by ER stress. KLHDC7B, a novel factor upregulated by ER stress, was significantly increased in response to wild-type but not mutant SubAB treatment.

\section{KLHDC7B is involved in SubAB-induced apoptosis}

As shown by RNA-seq analysis, KLHDC7B mRNA was most significantly stimulated by SubAB (Table 1). First, we tested if SubAB treatment increases KLHDC7B mRNA level using real-time, quantitative PCR (RT-qPCR). The RNA-seq data set was used to design the primer sequences (Table 2). The expression level of KLHDC7B mRNA was significantly increased in HeLa cells after $12 \mathrm{~h}$ of incubation with SubAB (Fig. 2A) In addition, we also found that SubAB increased KLHDC7B mRNA levels in the human colon cancer HCT116 cell line (Fig. S1).
Next, we evaluated the effect of KLHDC7B knockdown by siRNA on SubAB-induced apoptosis. Control or KLHDC7B siRNAtransfected HeLa cells were incubated with toxins for $24 \mathrm{~h}$. Then, we measured KLHDC7B mRNA levels by RT-qPCR. As shown in Fig. 2B, SubAB-stimulated KLHDC7B mRNA was significantly suppressed in KLHDC7B siRNA-transfected cells. Previous studies showed that SubAB-induced cell death was dependent on caspase activation-mediated apoptosis [12, 13]. In KLHDC7B-knockdown cells, SubAB-stimulated PARP cleavage, an apoptosis marker, was inhibited in comparison with the control siRNA-transfected cells, but not BiP cleavage (Fig. 2C, D). Next, we measured the effect of KLHDC7B knockdown on cell viability in the presence of SubAB. In control cells, SubAB decreased cell viability in a dose-dependent manner. Additionally, SubAB-induced cell death was attenuated in KLHDC7B-knockdown cells (Fig. 2E). As shown in Fig. $2 \mathrm{C}$ and $\mathrm{E}$, SubAB-induced changes in the conformation of Bax/Bak were significantly suppressed in KLHDC7B-knockdown cells, in comparison with control siRNA-transfected cells (Fig. 2F).

Brefeldin A (BFA) inhibits vesicle formation and trafficking between the ER and Golgi apparatus [31], thereby inhibiting SubAB-mediated BiP cleavage in the ER [17]. To evaluate the effect of BFA on SubAB-increased KLHDC7B, cells were incubated with 
A

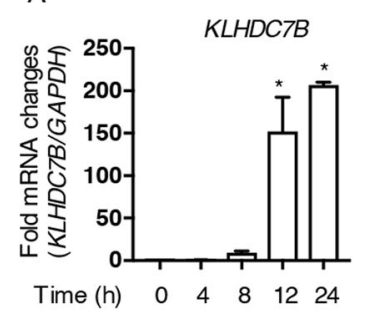

D

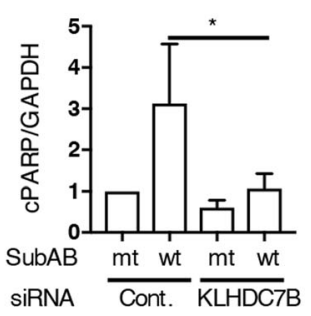

F

SiRNA NC KLHDC7B

SubAB mt wt mt wt kDa

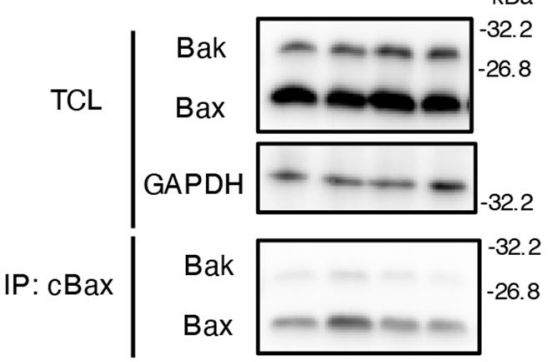

B

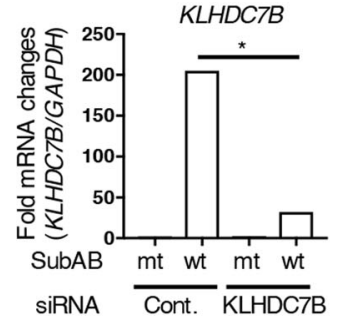

C

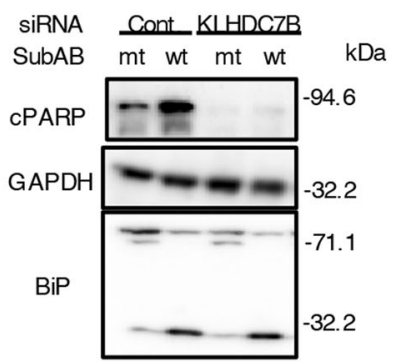

E

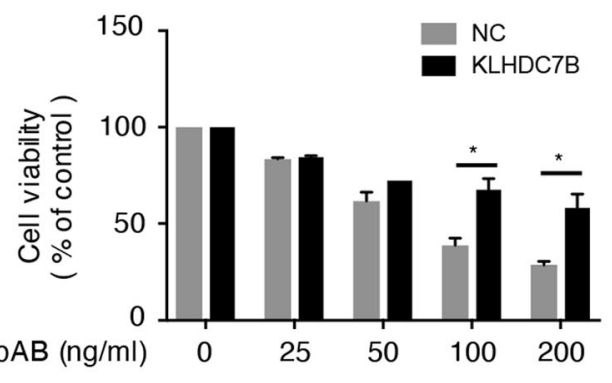

G

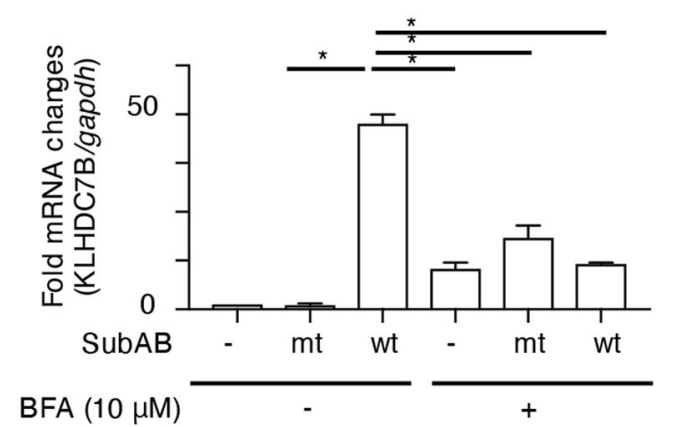

Fig. 2 SubAB induces KLHDC7B expression. A HeLa cells were incubated with $400 \mathrm{ng} \mathrm{mL}^{-1}$ of SubAB for the indicated duration. The $K L H D C 7 B$ mRNA levels were measured using RT-qPCR. GAPDH was used as the internal control. Data are presented as mean \pm standard deviation from three independent experiments. ${ }^{*} P<0.05$ versus untreated control cells. B Control (NC) or KLHDC7B siRNA-transfected HeLa cells were incubated for $24 \mathrm{~h}$ with $400 \mathrm{ng} \mathrm{mL}^{-1}$ of catalytically-inactive SubA ${ }_{S 272 A} B(\mathrm{mt})$ or SubAB (wt). The mRNA levels of $K L H D C 7 B$ were measured using RT-qPCR. GAPDH was used as the internal control. Data are presented as mean \pm standard deviation $(n=3)$. ${ }^{*} P<0.05$ versus mt SubAB-treated control cells. C Control (NC) or KLHDC7B siRNA-transfected cells were incubated for $16 \mathrm{~h} \mathrm{with} 400 \mathrm{ng} \mathrm{mL}{ }^{-1}$ of SubA $272 \mathrm{~A} B$ $(\mathrm{mt})$ or SubAB (wt). Immunoblotting with the antibodies was performed on cell lysates. GAPDH served as the loading control. D Densitometry was used to quantify the level of CPARP in HeLa cells. Data are presented as mean \pm standard deviation from three independent experiments. ${ }^{*} P<0.05$. E Control (NC) or KLHDC7B siRNA-transfected cells were incubated for $96 \mathrm{~h}$ with SubAB (wt). Cell viability was monitored using the Cell Counting Kit. Data are presented as mean \pm standard deviation from three independent experiments. ${ }^{*} P<0.05$. F The siRNA-transfected cells were incubated with $\mathrm{mt}$ or wt SubAB for $16 \mathrm{~h}$; cells were lysed and immunoprecipitated using conformationspecific anti-Bax (cBax) monoclonal antibodies. SDS-PAGE was used to analyze the immunocomplexes (IP) or total cell lysates (TCL), followed by immunoblotting with anti-Bax and anti-Bak antibodies. Data are mean values from two or more independent experiments. G Cells were incubated with $10 \mu \mathrm{M}$ of brefeldin A (BFA) with or without $400 \mathrm{ng} \mathrm{mL}^{-1}$ of SubA $\mathrm{S}_{5272 \mathrm{~A}} \mathrm{~B}(\mathrm{mt})$ or SubAB (wt) for $16 \mathrm{~h}$. The KLHDC7B mRNA levels were measured using RT-qPCR. GAPDH was used as the internal control. Data are presented as mean \pm standard deviation from three independent experiments. ${ }^{*} P<0.05$.

SubAB for $24 \mathrm{~h}$ with or without BFA. In the presence of BFA, SubAB-stimulated KLHDC7B mRNA was significantly suppressed; BFA alone increased KLHDC7B mRNA expression (Fig. 2G). Our results suggested that $E R$ stress due to SubAB-induced BiP cleavage increased KLHDC7B.

\section{Localization of FLAG-tagged KLHDC7B in HeLa cells}

We evaluated the effect of overexpression of FLAG-tagged KLHDC7B on SubAB-induced apoptosis. In FLAG-tagged KLHDC7Btransfected cells, PARP cleavage did not increase in the presence of SubAB after $8 \mathrm{~h}$ of incubation (Fig. 3A, B). The localization of
KLHDC7B has not been identified. To establish the subcellular distribution of KLHDC7B, we performed cell fractionation experiments using FLAG-tagged KLHDC7B-transfected HeLa cells, and investigated whether the subcellular localization of KLHDC7B changed in response to SubAB. We found that KLHDC7B localized to both the cytoplasmic and nuclear compartments, despite adding SubAB (Fig. 3C). To confirm the fractionation results, we stained FLAG-tagged KLHDC7B with immunofluorescence-labeled antibodies and used the DNA-binding fluorochrome DAPI. Consistent with the fractionation results, FLAG-tagged KLHDC7B (red) was localized to both the cytosolic and nuclear compartments, suggesting that 


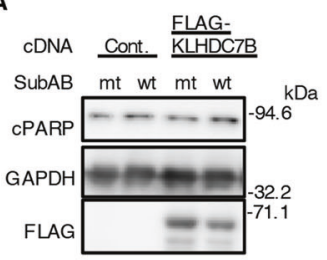

C

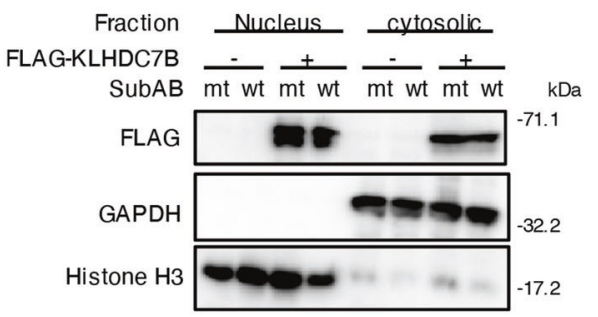

B

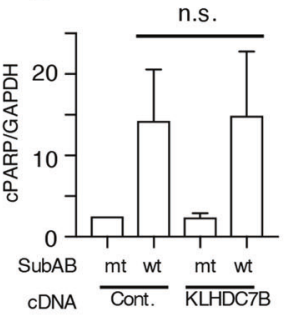

D

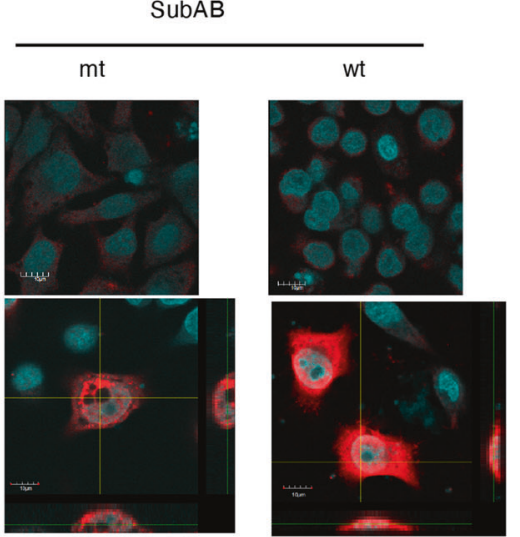

Fig. 3 KLHDC7B is localized to the nuclear and cytoplasmic compartments. A Control or FLAG-tagged KLHDC7B plasmid-transfected cells were incubated for $8 \mathrm{~h}$ with $400 \mathrm{ng} \mathrm{mL}^{-1}$ of SubA $\mathrm{S}_{\mathrm{S} 22 \mathrm{~A}} \mathrm{~B}(\mathrm{mt})$ or SubAB $(\mathrm{wt})$. Immunoblotting was performed for the cell lysates. GAPDH served as the loading control. B Densitometry was used to quantify the CPARP levels in HeLa cells. Data are presented as mean \pm standard deviation from three independent experiments. C, D Control or FLAG-tagged KLHDC7B plasmid-transfected cells were incubated for $16 \mathrm{~h}$ with $400 \mathrm{ng}$ $\mathrm{mL}^{-1}$ of SubA $\mathrm{S}_{272 \mathrm{~A}} \mathrm{~B}(\mathrm{mt})$ or SubAB (wt). Cells underwent fractionation with the Nuclear/Cytosol Fractionation Kit (WAKO). Histone 3 and GAPDH served as the loading controls for the nuclear and cytosolic fraction, respectively (C). Localization of FLAG-tagged KLHDC7B was visualized by immunofluorescence stain with anti-FLAG antibodies. Data are representative of three independent experiments.

the localization of FLAG-tagged KLHDC7B was not affected by SubAB (Fig. 3D).

\section{SubAB-induced activation of the ATF4/CHOP/CEBPB pathway regulates KLHDC7B expression}

Previous studies demonstrated that SubAB-stimulated ER stress activates ER stress sensor proteins, which subsequently activate transcription factors and induce several proteins [10$12,16,18,32,33]$. We attempted to identify the pathway that regulates SubAB-stimulated KLHDC7B expression. PERK is an essential ER stress sensor that plays a role in apoptosis induced by SubAB [12]. SubAB-induced increase in KLHDC7B mRNA expression level was significantly attenuated in PERK-knockdown cells (Fig. 4A). We examined the effect of PERK-regulated transcription factors (e.g., ATF4, CHOP, and CEBPB) on KLHDC7B expression. As shown in Fig. 4B, knockdown of ATF4 and CHOP by siRNA significantly inhibited SubAB-stimulated KLHDC7B expression, which was partially suppressed in CEBPB-knockdown cells.

Next, we determined the order of ATF4, CHOP, and CEBPB in SubAB-stimulated KLHDC7B expression. In ATF4-knockdown cells, SubAB-induced increase in CHOP and CEBPB mRNA levels were attenuated. Although SubAB-induced increase in CHOP mRNA expression level was not attenuated in CEBPB-knockdown cells, knockdown of $\mathrm{CHOP}$ suppressed SubAB-induced increase in CEBPB mRNA expression levels (Fig. 4C). We further investigated whether KLHDC7B knockdown affects SubAB-induced activation of transcription factors, e.g., CHOP, ATF4. In KLHDC7B-knockdown cells, SubAB-induced increase in ATF4 mRNA expression level was not changed, but CHOP mRNA expression level was significantly decreased (Fig. 4D). Consistent with the RT-qPCR results, the Western blotting analysis showed that SubAB-induced increase in CHOP expression was attenuated in KLHDC7B-knockdown cells
(Fig. 4E), suggesting that SubAB-induced KLHDC7B expression is regulated by the PERK/ATF4/CHOP/CEBPB signaling pathway, and that the expressed KLHDC7B controls CHOP.

Identification of KLHDC7B-regulating factors in SubAB-treated cells by RNA-seq and differential expression analysis

The function of KLHDC7B remains unknown. To explore the role of SubAB-stimulated KLHDC7B, we performed RNA-seq analysis using control or KLHDC7B siRNA-transfected cells with mutant or wild-type SubAB. A total of 100 million paired end reads, with an average of 50 million reads per sample, were mapped on a reference human genome UCSC GRCh37/hg19 (Table 3). A heat map analysis indicated that wild-type SubAB treatment resulted in different gene expression (enriched or depleted changes of host transcripts) between the control and KLHDC7B siRNA-transfected cells (Fig. 5A). The analysis of biological processes and cellular components suggested SubAB increased (i) intrinsic apoptotic signaling pathway, (ii) IRE1-mediated, unfolded-protein response, and (iii) regulation of response to $E R$ stress, which were significantly suppressed in KLHDC7B-knockdown cells (Fig. 5B). Similar to the results from the RNA-seq data, RT-qPCR analysis showed that SubAB-induced increase in the mRNA levels (e.g., CAPN5, NUPR1, CHOP, GADD45A, ATP8B3, HERPUD1, HNF4A, GADD34, CRELD2, PDIA4) were significantly attenuated in KLHDC7Bknockdown cells (Fig. 5D).

KLHDC7B regulates the expression of HRK, which is essential for SubAB-induced apoptosis

To investigate how SubAB-induced increase in KLHDC7B regulates apoptosis, we searched for the apoptosis-related genes that were increased or decreased by SubAB in control cells, and showed an opposite response in KLHDC7B-knockdown cells. In RNA-seq analysis, we found that a gene encoding for the pro-apoptotic 
A

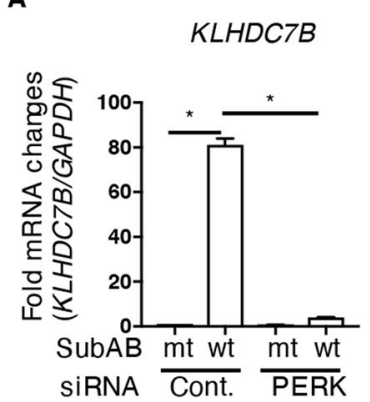

C

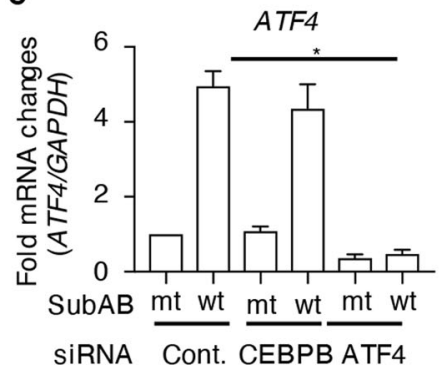

B

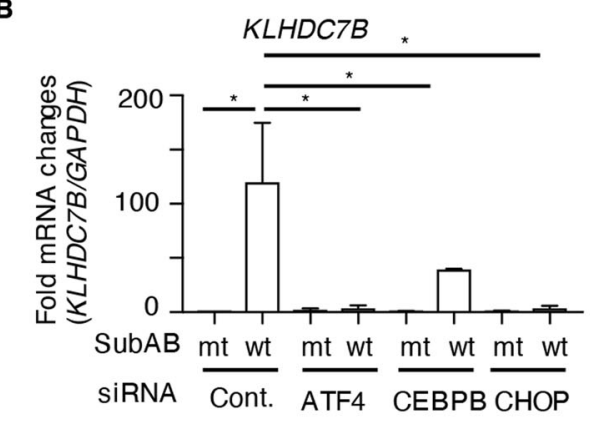

D

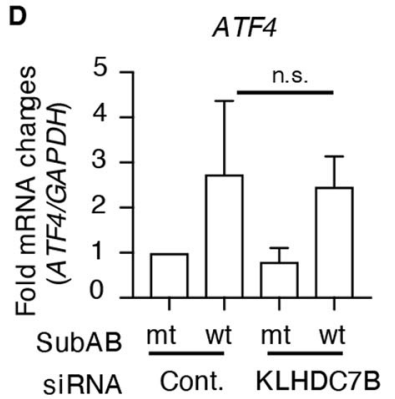

E

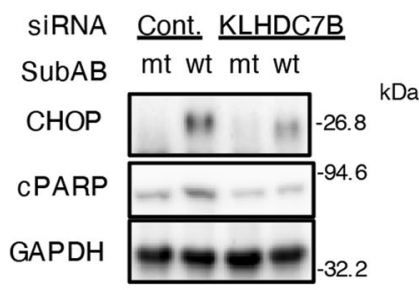

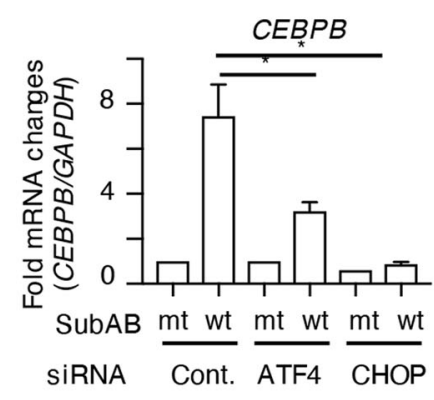

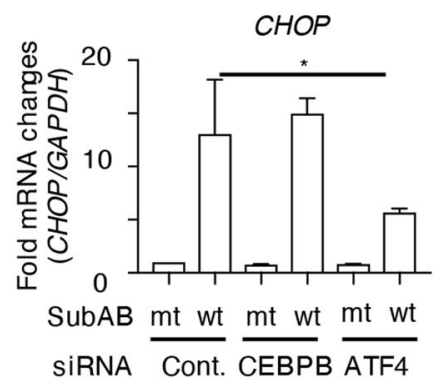

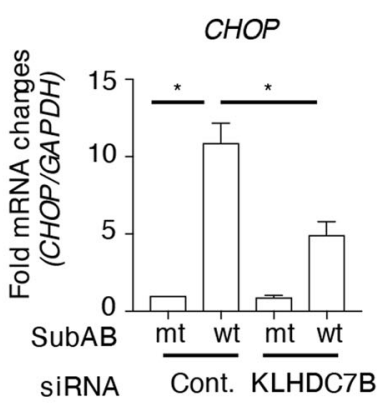

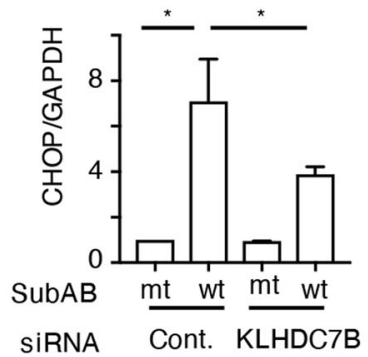

Fig. 4 KLHDC7B was regulated by PERK/ATF4/CEBPB. A-D siRNA-transfected cells were incubated for $16 \mathrm{~h}^{\text {with }} 400 \mathrm{ng} \mathrm{mL}^{-1}$ of SubA $\mathrm{S}_{272 \mathrm{~A}} \mathrm{~B}$ (mt) or SubAB (wt). The KLHDC7B, ATF4, CEBPB, and CHOP mRNA levels were measured using RT-qPCR. GAPDH was used as the internal control. Data are presented as mean \pm standard deviation $(n=3)$. ${ }^{*} p<0.05$. E Control (NC) or KLHDC7B siRNA-transfected cells were incubated for $16 \mathrm{~h}$ with $400 \mathrm{ng} \mathrm{mL}^{-1}$ of SubA $\mathrm{S}_{272 \mathrm{~A}} \mathrm{~B}(\mathrm{mt})$ or SubAB (wt). Immunoblotting was performed on the cell lysates and GAPDH served as the loading control. Densitometry was used to quantify the CHOP level in HeLa cells. Data are presented as mean \pm standard deviation from three independent experiments. ${ }^{*} p<0.05$.

protein HRK was significantly increased by SubAB in control cells, but decreased by SubAB in KLHDC7B-knockdown cells. Using RTqPCR analysis, in control cells, SubAB increased HRK mRNA levels, whereas decreased it in KLHDC7B-knockdown cells (Fig. 6A). We found that, similar to SubAB, a general ER stress inducer, tunicamycin (TM), also increased mRNA levels of CHOP, KLHDC7B, and HRK expression (Fig. S2). Although we examined the expression level of HRK by Western blotting using commercial
anti-HRK antibodies (i.e., \#NBP1-59447, Novus Biologicals; \#PRS3771, Sigma Aldrich), we did not detect a specific human $\mathrm{HRK}$ band. HRK interacts with $\mathrm{BCl}-2$ and $\mathrm{Bcl}-\mathrm{xL}$, which are members of the anti-apoptotic Bcl protein family that inhibits HRK-induced cell death [34]. Previous studies showed that SubAB triggered changes in the conformation of Bax and Bak, which form a complex on the mitochondria $[12,13,18,35]$. Next, we investigated the effect of HRK knockdown on conformational 
Table 3. Mapped data stats of RNA-seq analysis.

\begin{tabular}{lllll} 
Library & Total reads & Read length (bp) & Mapped reads & Mapped reads (\%) \\
\hline Cont._mt SubAB & $53,417,956$ & 101 & $52,299,104$ & 97.91 \\
\hline Cont._wt SubAB & $51,471,614$ & 101 & $50,456,121$ & 98.03 \\
\hline KLHDC7B siRNA_mt SubAB & $55,435,614$ & 101 & $54,409,690$ & 98.15 \\
\hline KLHDC7B siRNA_wt SubAB & $49,729,780$ & 101 & $48,569,520$ & 97.67
\end{tabular}

changes in Bax/Bak induced by SubAB using immunoprecipitation. The HRK mRNA level was significantly lower by HRK siRNA, which suppressed SubAB-induced increase in HRK mRNA expression levels (Fig. S3). Furthermore, SubAB-induced conformational changes in Bax/Bak were attenuated in HRK-knockdown cells (Fig. 6B). Consistent with these results, SubAB-induced PARP cleavage was significantly suppressed in HRK-knockdown compared to control cells (Fig. $6 \mathrm{C}$ ). Further, in control cells, SubAB decreased cell viability after $96 \mathrm{~h}$ of incubation, while SubABinduced decreased cell viability was inhibited in the HRKknockdown cells (Fig. 6D). In cells overexpressing HRK, SubABinduced PARP cleavage was enhanced (Fig. 6E). These data showed that increased HRK expression alone enhanced PARP cleavage, and this effect was enhanced with SubAB. Our findings suggest that SubAB-induced apoptosis is dependent on the mitochondria, and involves KLHDC7B-mediated HRK expression, followed by Bak/Bax-conformational changes.

\section{DISCUSSION}

To elucidate the mechanism of SubAB-induced apoptotic signaling, we performed RNA-seq analysis. Compared with inactivated SubAB mt, SubAB wt induced significant upregulation of 452 genes and downregulation of 271 genes (Fig. 1A). As shown in the GO term enrichment analysis (Fig. 1B), SubAB-induced cleavage of BiP stimulated multiple ER responses, including upregulation of ER stress markers, e.g., ATF4, CHOP genes (Fig. 4C). A recent study in HeLa cells reported that chemical ER stress inducers stimulated ATF4, followed by induction of mRNA expression of the inflammasome component, NLR Family Pyrin Domain Containing 1 (NLRP1), in HeLa cells [36]. The study demonstrated that the IRE1 and PERK pathways were important for the induction of NLRP1 mRNA expression and that ATF4, but not XBP-1, was critical for the induction. They also suggested that factors other than XBP-1 were involved in the IRE1 pathway. In our RNA-seq results, we observed that the inflammasome-related genes were not increased in SubAB-treated HeLa cells. These findings suggest that the contribution of different activated pathways may differ between chemical ER stress inducers and SubAB.

Using siRNA-knockdown cells, we found that KLHDC7B is a novel cell-death mediator. Although the function of KLHDC7B remains unknown, increased expression of KLHDC7B was observed in various tumors. However, the expression level was not always higher than that observed in non-tumor tissues [19]. In human breast cancer MCF cells, proliferation was decreased in KLHDC7B-overexpressing cells, and increased in KLHDC7B-knockdown cells [37]. In contrast, in the current study, we demonstrated that SubAB-induced apoptosis was significantly decreased in KLHDC7B-knockdown HeLa cells, suggesting that KLHDC7B was associated with cell proliferation, but the role of KLHDC7B may differ, depending on the tissue or stimulator.

SubAB-induced ER stress stimulated dramatic upregulation of KLHDC7B mRNA expression. The promoter region of KLHDC7B is a rarely hypermethylated gene in breast cancer cell lines [21]. We investigated whether the hypermethylation of the KLHDC7B promoter in HeLa cells participated in the expression by SubABinduced ER stress. DNA methyltransferase inhibitor RG108 and 5Aza-2'-deoxycytidine (5-Aza) did not affect SubAB-increased KLHDC7B mRNA expression levels or SubAB-induced PARP cleavage (Fig. S4A and B), suggesting that promoter methylation was not involved in KLHDC7B expression in HeLa cells. Our results suggest that SubAB-stimulated KLHDC7B mRNA expression level was regulated by increased ATF4 directly or indirectly by a pathway involved in CHOP or CEBPB. Interestingly, KLHDC7B knockdown suppressed SubAB-increased CHOP expression, suggesting that KLHDC7B is related to CHOP expression.

SubAB-induced apoptosis was mediated by conformational changes and effects of oligomerization of Bax/Bak on mitochondria, cytochrome $c$ release, and caspase activation [13]. The pathway was suppressed by the anti-apoptotic Bcl-2 family member Bcl-xLoverexpressing cells [18]. These findings were consistent with the observation that $\mathrm{Bcl}-\mathrm{xL}$ blocks the physical encounter of Bax and Bak on mitochondria, which is necessary for subsequent apoptosis. However, the molecular mechanisms leading to Bax/Bak conformational changes remain poorly defined. Besides $\mathrm{Bcl}-\mathrm{xL}$, previous studies showed that several factors, including Bid translocation from the cytosol to mitochondria [38], Bif-1 [39], apoptosisassociated speck-like protein (ASC) [40], Ku70 [41], 14-3-3 theta [42], and p53 [43], have participated in the regulation of Bax/Bax activity. Here, we identified by RNA-seq analysis data that SubAB induced an increase in HRK, regulated by KLHDC7B, involved in the generation of conformationally different Bax/Bak, followed by apoptosis. HRK has a $\mathrm{BH} 3$ region of the $\mathrm{Bcl}-2$ member and controls apoptosis by inhibiting the pathway with anti-apoptotic BCl-2 and $\mathrm{BCl}-\mathrm{xL}$ [34]. Previous studies reported that HRK participates in cell death, which is suppressed in $\mathrm{BCl}-2$ - and/or $\mathrm{BCl}$-xL-overexpressing cells $[44,45]$. Knockdown of HRK expression by siRNA significantly suppressed SubAB-induced apoptotic pathway and increased cell viability, suggesting that HRK is an essential apoptotic factor. These findings suggest that SubAB-induced increase in HRK inhibited $\mathrm{BCl}$ $\mathrm{xL}$ and/or $\mathrm{BCl}-2$ activity, followed by promotion of Bax/Bak conformational changes, resulting in cell death.

Previous reports showed that HRK mRNA in human tissues was highly expressed in spleen and bone marrow, and expressed at low levels in kidney, liver, lung, and brain [34]. Analysis of the HRK promoter region showed that HRK transcription was controlled by c-Jun, ATF2 [46, 47], and transcription repressor DREAM [48]. A recent study showed that Yes-associated protein (YAP)-knockdown increased HRK expression in neuroblastoma, suggesting that YAP is a suppressor of HRK expression [49]. ATrich interactive domain-containing protein $1 \mathrm{~A}$ (ARID1A) is a subunit of the SWI/SNF chromatin remodeling complexes which bind DNA. Knockdown of ARID1A decreased HRK expression without direct binding to the HRK gene in gastric cancer cells [50]. Therefore, HRK expression is regulated by various transcription factors. KLHDC7B knockdown inhibited the expression of HRK transcription by SubAB. Although we could not detect a direct interaction between KLHDC7B and HRK promoter region by a ChIP assay, KLHDC7B might be a novel regulator as a transcription factor of HRK expression.

Figure $6 \mathrm{~F}$ summarizes the proposed model of SubAB-increased KLHDC7B mediated cell death pathway. SubAB-activated PERK increased expression levels of ATF4 and CHOP, followed by upregulation of CEBPB. These transcription factors induced KLHDC7B expression, which upregulated HRK expression. HRK caused conformational changes of Bak/Bax, thereby causing cell death. 

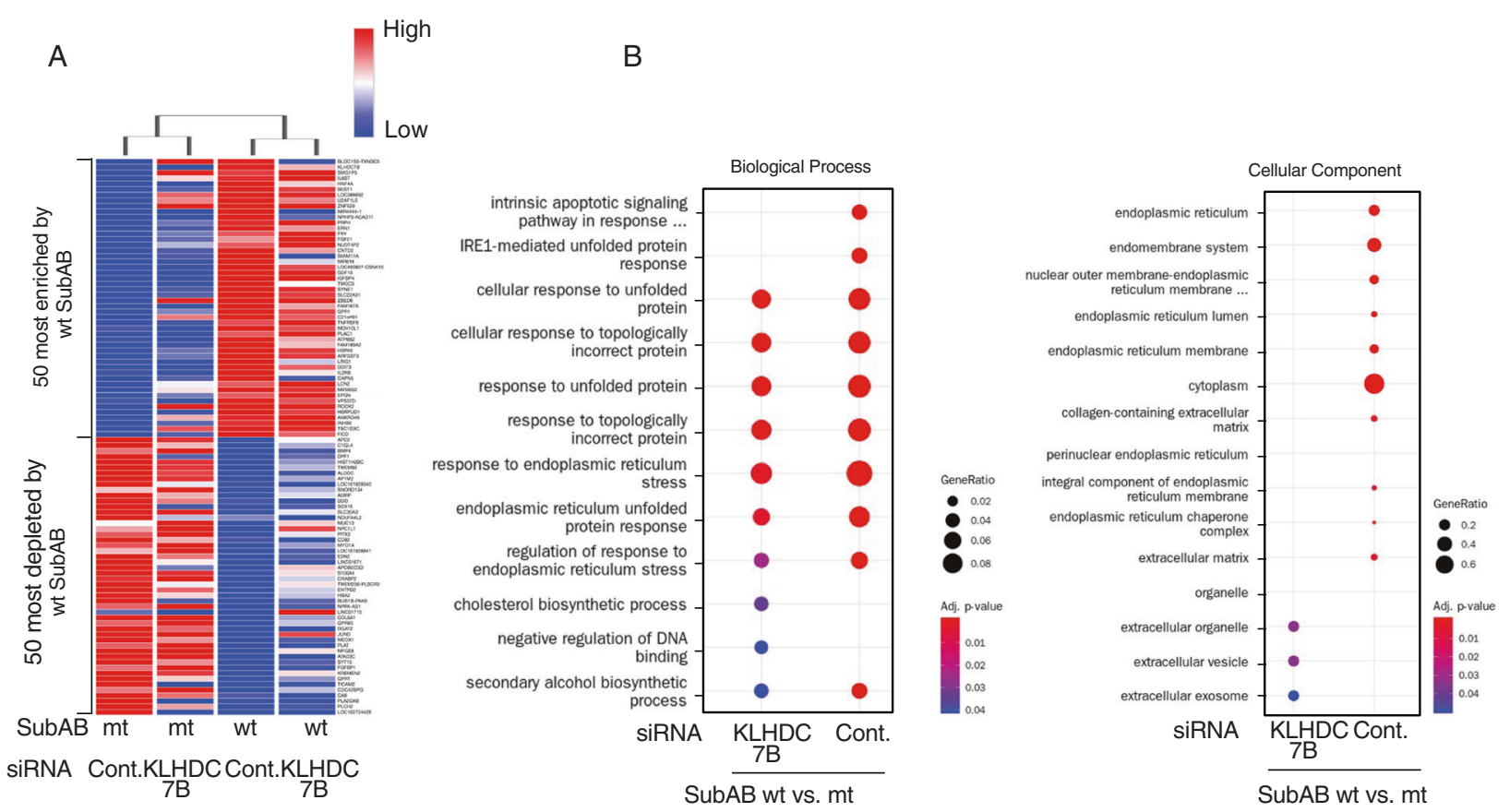

C
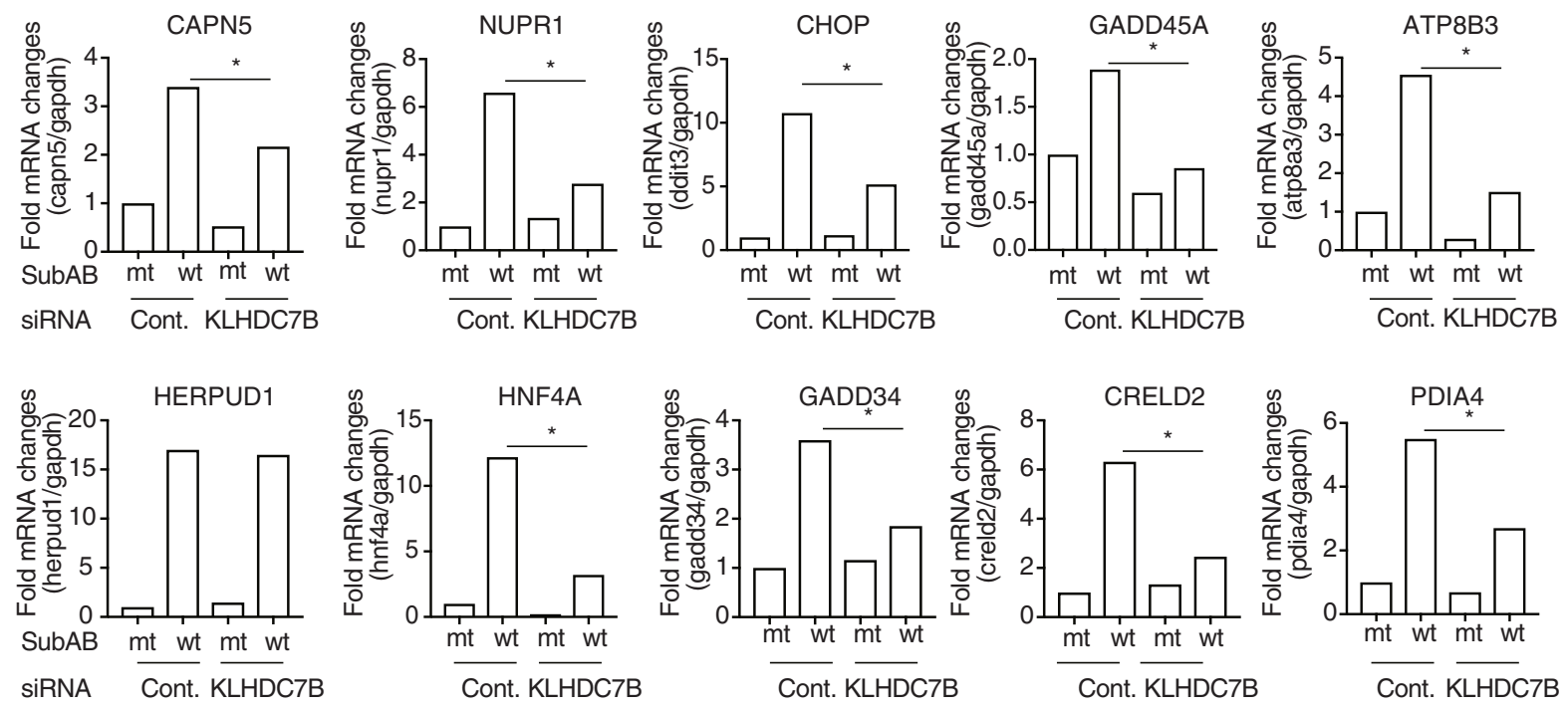

Fig. 5 RNA-seq analysis between control and KLHDC7B knockdown cells. Control or KLHDC7B siRNA-transfected cells were incubated for $16 \mathrm{~h}$ with $400 \mathrm{ng} \mathrm{mL}^{-1}$ of SubA ${ }_{S 272 \mathrm{~A}} \mathrm{~B}(\mathrm{mt})$ or SubAB (wt), and then purified mRNA. These mRNAs were investigated by RNA-seq analysis. A Heat map of most enriched or depleted 50 differentially expressed genes. B The RNA-seq results are summarized in two categories: biological process and cellular components. C The mRNA levels were measured using RT-qPCR. GAPDH served as the internal control and $C H O P$ as the positive control. Data are representative of two independent experiments. ${ }^{*} p<0.05$.

\section{MATERIALS AND METHODS}

\section{Preparation of subtilase cytotoxin}

Recombinant, His-tagged, wild-type subtilase cytotoxin (wt SubAB) or catalytically-inactivated mutant SubA (S272A)B ( $m$ t SubAB) was prepared in Escherichia coli (E. coli) BL21, followed by purification with $\mathrm{Ni}$ nitrilotriacetic acid (NTA) agarose (Qiagen, Hilden, Germany), similar to the previously published procedure [14].

\section{Antibodies and other reagents}

Antibodies targeting cleaved PARP (CPARP) (\#5625), CHOP/GADD153 (\#2895), PERK (\#3192), Bax (\#2772), and Bak (\#3814) were purchased from Cell Signaling Technology (Beverly, MA, USA). Mouse monoclonal antibodies against
BiP/GRP78 (\#610978) and Bax (\#610982) were purchased from BD Biosciences (Franklin Lakes, NJ, USA). Anti-FLAG (\#014-22383) monoclonal antibody was purchased from Fujifilm WAKO Pure Chemical Corp (Tokyo, Japan). Anti-FLAG (\#66008-3-lg) and anti-GAPDH antibodies (\#10494-1-AP) were purchased from Proteintech (Wuhan, China). Myc-DDK-tagged HRK expression plasmid was purchased from OriGene (Rockville, MD, USA).

\section{Purification of RNA for RNA-seq}

HeLa cells were cultured in 6-well plates for $16 \mathrm{~h}$ with $\mathrm{mt}$ or wt SubAB (400 $\mathrm{ng} \mathrm{mL}^{-1}$ ). Total RNA was purified using ISOGEN II (Nippon Gene Co., Ltd., Toyama, Japan). The paired end RNA-seq libraries were constructed using TruSeq Strand mRNA LT Sample Prep Kit and sequenced using 
A

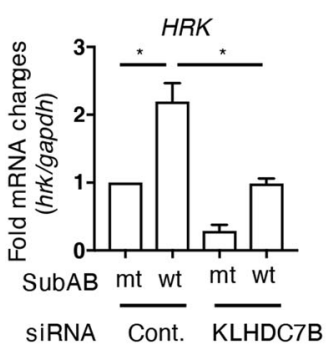

C

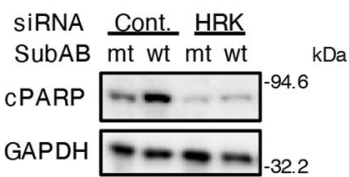

E

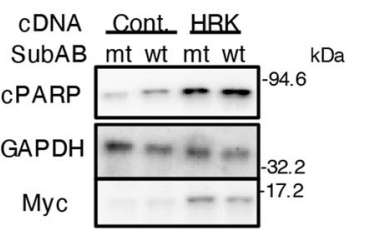

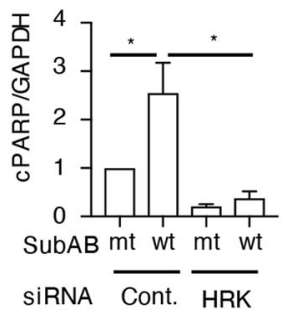

$\mathbf{F}$<smiles></smiles>
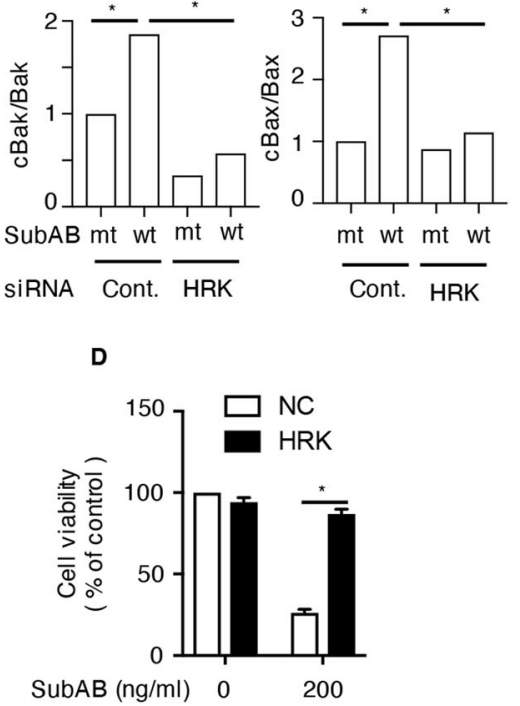

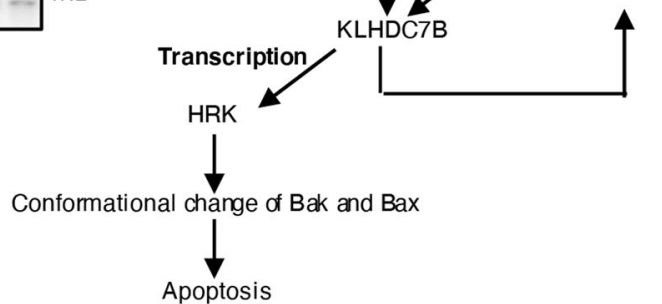

Fig. 6 RNA-seq analysis of control and KLHDC7B knockdown cells. A The siRNA-transfected cells were incubated for 20-24 $\mathrm{h}$ with mt or wt SubAB. Then, the HRK mRNA levels were measured using RT-qPCR. GAPDH was used as the internal control. Data are presented as mean \pm standard deviation $(n=3) .{ }^{*} p<0.05$. B CHAPS buffer extracts from the siRNA-transfected cells were treated with mt or wt SubAB, and proteins were co-immunoprecipitated with conformation-specific, anti-active Bax monoclonal antibody (cBax). SDS-PAGE was used to analyze the immunocomplexes and total cell lysates $(\mathrm{TCL})$, followed by immunoblotting with antibodies. A representative blot of two independent experiments is shown. Conformationally-changed Bax and co-precipitated Bak were quantified using a densitometer (right panel). C The siRNA-transfected cells were incubated for 20-24 h with $\mathrm{mt}$ or wt SubAB. Then, the cell lysates underwent immunoblotting with the indicated antibodies. GAPDH served as the loading control. Densitometry was used to quantify the cPARP level in HeLa cells. Data are presented as mean \pm standard deviation from three independent experiments. ${ }^{*} p<0.05$. D siRNA-transfected cells were incubated for $96 \mathrm{~h}$ with mt or wt SubAB, and observed for cell viability using the Cell Counting Kit. Data are presented as mean \pm standard deviation from three independent experiments. ${ }^{*} p<0.05$. E The cDNA-transfected cells were incubated for $16 \mathrm{~h}$ with $\mathrm{mt}$ or wt SubAB. Then, the cell lysates underwent immunoblotting with the antibodies. GAPDH served as the loading control. Densitometry was used to quantify the cPARP level in HeLa cells. Data are presented as mean \pm standard deviation from three independent experiments. ${ }^{*} p<0.05$. F The proposed model of SubAB-increased KLHDC7B regulates apoptotic cell death pathway via HRK expression.

Illumine HiSeq 2500. Therefore, 101 pair length reads were obtained using Genewitz Inc. (South Plainfield, NJ, USA) or Macrogen Japan (Kyoto, Japan).

\section{Differentially expressed genes and functional enrichment}

Read counts and normalization values are determined by the transcript length and depth of coverage, and therefore indicate gene expression profiles. Fragments per kilobase of transcript per million mapped reads (FPKM) values or reads per kilobase of transcript per million mapped reads (RPKM) are often used as normalization values. The gene expression levels were compared between controls and toxin-treated cells to identify significant differentially expressed genes (DEGs). Among the DEGs, we selected those that had a greater than 2 -fold difference and $p$-value $<0.05$ compared to control cells. In addition, these DEGs were used for functional enrichment analysis using Gene Ontology (GO) and Kyoto Encyclopedia of Genes and Genomics (KEGG) pathway.

\section{Cell culture and gene transfection}

HeLa cells (RIKEN, RCB0007) were cultured in RPMl1640 medium (SigmaAldrich, St. Louis, MO, USA). The medium contained $10 \%$ fetal calf serum and penicillin-streptomycin solution (Sigma-Aldrich). The cells were plated with a medium containing 10\% FCS. RNA interference-mediated gene knockdown was performed using validated Qiagen HP small-interfering RNAs (siRNAs) for PERK (SI02223718) and C/EBPB (SI02777292). ATF4 siRNA was designed and validated, as described previously [51]. CHOP and KLHDC7B siRNAs were purchased from Santa Cruz Biotechnology (Santa Cruz, CA, USA). HRK siRNA 
(s194952) was purchased from Ambion (Austin, TX, USA). Cells were transfected with 50-100 nM of siRNAs for $48 \mathrm{~h}$ using Lipofectamine ${ }^{\mathrm{TM}}$ RNAiMax transfection reagent (Invitrogen, Carlsbad, CA, USA), as per the manufacturer's instructions. Protein knockdown was confirmed by RT-qPCR or immunoblotting with the aforementioned antibodies.

\section{Construction of FLAG-tagged KLHDC7B}

ISOGEN II was used to purify the total RNA from SubAB-treated HeLa cells. Then, PrimeSTAR HS DNA Polymerase kit (TaKaRa Bio Inc., Shiga, Japan) was used to amplify CDNA in a 25- $\mu \mathrm{L}$ PCR mixture, as per the manufacturer's instructions. The PCR conditions for KLHDC7B included 30 cycles of $98^{\circ} \mathrm{C}$ for $15 \mathrm{~s}, 60^{\circ} \mathrm{C}$ for $10 \mathrm{~s}$, and $72^{\circ} \mathrm{C}$ for $3 \mathrm{~min}$. Table 2 summarizes the primers used for PCR. The PCR products were ligated into the Not1 and Sal1 sites of FLAG5 a expression vector (Sigma-Aldrich) using In-Fusion HD Cloning Kit (TaKaRa Bio Inc.). The FLAG-tagged human KLHDC7B plasmid was sequenced and transfected into HeLa cells using Polyethylenimine Max (Polysciences Inc. Warrington, PA, USA) in OPTI-MEM I reduced-serum medium (ThermoFisher Scientific, Waltham, MA, USA).

\section{Real-time qPCR}

PrimeScript ${ }^{\text {TM }}$ RT Master Mix (TaKaRa Bio Inc.) was used to amplify the CDNA in $10 \mu \mathrm{L}$ of PCR mixture, as per the manufacturer's instructions. KOD SYBER qPCR Mix (Toyobo, Osaka, Japan) and ABI Prism 7000 (PerkinElmer Life Sciences, Boston, MA, USA) were used to perform RT-qPCR. Table 2 shows the primers used for PCR. Relative expression levels were normalized to GAPDH and calibrated to the respective controls.

\section{Immunoblot analysis}

Cell proteins were separated using SDS-PAGE and transferred to PVDF membranes, followed by incubation with the aforementioned primary antibodies. Horseradish peroxidase-labeled goat anti-mouse or anti-rabbit secondary antibodies (R\&D systems, Abingdon, UK) were used for detection, followed by enhanced chemiluminescence (EzWestLumi One; ATTO Corp., Tokyo, Japan). Las 1000 (FUJIFILM) was used to visualize the bands and the Image Gauge software (FUJIFILM) was used for densitometric analysis of the scanned blots. The protein levels were normalized to GAPDH.

\section{Immunoprecipitation of conformationally-changed Bak/Bax}

The siRNA-transfected cells $\left(3 \times 10^{5}\right.$ cells/ 12 -well plate) were incubated with $400 \mathrm{ng} / \mathrm{mL}$ of SubAB or mt SubAB for $12 \mathrm{~h}$ at $37^{\circ} \mathrm{C}$. Coimmunoprecipitation of conformationally-changed Bax or Bak followed the methods described in previous studies [12].

\section{Immunofluorescence confocal microscopy}

Immunofluorescence analysis was performed as described previously [8, 12]. Briefly, FLAG-tagged KLHDC7B-transfected HeLa cells $\left(3 \times 10^{5}\right.$ cells/12-well plate) on glass (Matsunami Glass Co. Ltd., Osaka, Japan) were incubated with $400 \mathrm{ng} \mathrm{mL}^{-1}$ of SubAB or mt SubAB. Cells were fixed with $4 \%$ paraformaldehyde (PFA), rinsed three times with PBS, and incubated with the blocking buffer (5\% goat serum and $0.3 \%$ Triton X-100 in PBS) at room temperature for $1 \mathrm{~h}$. Cells were further incubated with anti-FLAG antibodies (\#014-22383, WAKO) in $0.4 \%$ BSA/PBS buffer at $4{ }^{\circ} \mathrm{C}$ overnight, washed twice with PBS, and incubated with anti-mouse Cy3 (\#ab97035, Abcam, Cambridge, UK) antibodies at room temperature for $1 \mathrm{~h}$ in the dark.

After the cells were washed with PBS, they were mounted on glass slides using Prolong Gold Antifade reagent with DAPI (ThermoFisher Scientific). FV10i-LIV confocal microscopy (Olympus, Tokyo, Japan) was used to visualize the stained cells and the images were arranged with Adobe Photoshop CS4.

\section{Statistics}

Student's $t$-test was used for the densitometric analysis and analysis of RT-qPCR assays using the Graphpad Prism software (Graphpad Inc., San Diego, CA, USA). $P$-values $<0.05$ were considered to indicate statistical significance.

\section{DATA AVAILABILITY}

The raw reads data of RNAseq in this study are available in DNA Data Bank of Japan (DDBJ) under the accession number DRA012635.

\section{REFERENCES}

1. Scheutz F. Taxonomy meets public health: the case of shiga toxin-producing Escherichia coli. Microbiol Spectr 2014, 2.3.09.

2. Karmali MA. Prospects for preventing serious systemic toxemic complications of Shiga toxin-producing Escherichia coli infections using Shiga toxin receptor analogues. J Infect Dis. 2004;189:355-9.

3. Paton AW, Srimanote P, Talbot UM, Wang H, Paton JC. A new family of potent AB (5) cytotoxins produced by Shiga toxigenic Escherichia coli. J Exp Med. 2004;200:35-46.

4. Byres E, Paton AW, Paton JC, Lofling JC, Smith DF, Wilce MC, et al. Incorporation of a non-human glycan mediates human susceptibility to a bacterial toxin. Nature. 2008;456:648-52.

5. Yahiro K, Satoh M, Morinaga N, Tsutsuki H, Ogura K, Nagasawa S, et al. Identification of subtilase cytotoxin (SubAB) receptors whose signaling, in association with SubAB-induced BiP cleavage, is responsible for apoptosis in HeLa cells. Infect Immun. 2011;79:617-27.

6. Yamaji T, Hanamatsu H, Sekizuka T, Kuroda M, Iwasaki N, Ohnishi M, et al. A CRISPR screen using subtilase cytotoxin identifies SLC39A9 as a glycan-regulating factor. iScience. 2019;15:407-20.

7. Chong DC, Paton JC, Thorpe CM, Paton AW. Clathrin-dependent trafficking of subtilase cytotoxin, a novel $\mathrm{AB} 5$ toxin that targets the endoplasmic reticulum chaperone BiP. Cell Microbiol. 2008;10:795-806.

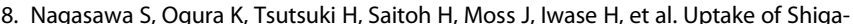
toxigenic Escherichia coli SubAB by HeLa cells requires an actin- and lipid raftdependent pathway. Cell Microbiol. 2014;16:1582-601.

9. Smith RD, Willett R, Kudlyk T, Pokrovskaya I, Paton AW, Paton JC, et al. The COG complex, Rab6 and COPI define a novel Golgi retrograde trafficking pathway that is exploited by SubAB toxin. Traffic. 2009;10:1502-17.

10. Tsutsuki H, Zhang T, Harada A, Rahman A, Ono K, Yahiro K, et al. Involvement of protein disulfide isomerase in subtilase cytotoxin-induced cell death in HeLa cells. Biochem Biophys Res Commun. 2020;525:1068-73. https://doi.org/10.1016/ j.bbrc.2020.03.008.

11. Wolfson JJ, May KL, Thorpe CM, Jandhyala DM, Paton JC, Paton AW. Subtilase cytotoxin activates PERK, IRE1 and ATF6 endoplasmic reticulum stress-signalling pathways. Cell Microbiol. 2008;10:1775-86.

12. Yahiro K, Tsutsuki H, Ogura K, Nagasawa S, Moss J, Noda M. Regulation of subtilase cytotoxin-induced cell death by an RNA-dependent protein kinase-like endoplasmic reticulum kinase-dependent proteasome pathway in HeLa cells. Infect Immun. 2012;80:1803-14.

13. Yahiro K, Morinaga N, Moss J, Noda M. Subtilase cytotoxin induces apoptosis in HeLa cells by mitochondrial permeabilization via activation of Bax/Bak, independent of C/EBF-homologue protein (CHOP), Ire1alpha or JNK signaling. Micro Pathog. 2010;49:153-63.

14. Morinaga N, Yahiro K, Matsuura G, Watanabe M, Nomura F, Moss J, et al. Two distinct cytotoxic activities of subtilase cytotoxin produced by shiga-toxigenic Escherichia coli. Infect Immun. 2007;75:488-96.

15. Tsutsuki H, Yahiro K, Suzuki K, Suto A, Ogura K, Nagasawa S, et al. Subtilase cytotoxin enhances Escherichia coli survival in macrophages by suppression of nitric oxide production through the inhibition of NF-kappa B activation. Infect Immun. 2012;80:3939-51.

16. Tsutsuki H, Yahiro K, Ogura K, Ichimura K, lyoda S, Ohnishi M, et al. Subtilase cytotoxin produced by locus of enterocyte effacement-negative shiga-toxigenic Escherichia coli induces stress granule formation. Cell Microbiol. 2016;18:1024-40.

17. Yahiro K, Ogura K, Goto $Y$, lyoda S, Kobayashi T, Takeuchi H, et al. Subtilase cytotoxin induces a novel form of Lipocalin 2, which promotes Shiga-toxigenic Escherichia coli survival. Sci Rep. 2020;10:18943.

18. Yahiro K, Nagasawa S, Ichimura K, Takeuchi H, Ogura K, Tsutsuki H, et al. Mechanism of inhibition of Shiga-toxigenic Escherichia coli SubAB cytotoxicity by steroids and diacylglycerol analogues. Cell Death Disco. 2018;4:22.

19. Martin-Pardillos A, Cajal SRY. Characterization of Kelch domain-containing protein $7 \mathrm{~B}$ in breast tumours and breast cancer cell lines. Oncol Lett. 2019;18:2853-60.

20. Zhang G, Fan E, Yue G, Zhong Q, Shuai $Y$, Wu M, et al. Five genes as a novel signature for predicting the prognosis of patients with laryngeal cancer. J Cell Biochem. 2019;121:3804-13.

21. Kim TW, Kim YJ, Lee HJ, Min SY, Kang HS, Kim SJ. Hs.137007 is a novel epigenetic marker hypermethylated and up-regulated in breast cancer. Int J Oncol. 2010;36:1105-11.

22. Xue F, Cooley L. Kelch encodes a component of intercellular bridges in Drosophila egg chambers. Cell. 1993;72:681-93.

23. Gupta VA, Beggs AH. Kelch proteins: emerging roles in skeletal muscle development and diseases. Skelet Muscle. 2014;4:11.

24. Yamaguchi H, Wang HG. CHOP is involved in endoplasmic reticulum stressinduced apoptosis by enhancing DR5 expression in human carcinoma cells. J Biol Chem. 2004;279:45495-502. 
25. Lindenmeyer MT, Rastaldi MP, Ikehata M, Neusser MA, Kretzler M, Cohen CD, et al. Proteinuria and hyperglycemia induce endoplasmic reticulum stress. J Am Soc Nephrol. 2008;19:2225-36.

26. Nomura Y, Hirata Y, Kiuchi K, Oh-Hashi K. Translational and post-translational regulation of mouse cation transport regulator homolog 1. Sci Rep. 2016;6:28016.

27. Li D, Zhang $H$, Zhong Y. Hepatic GDF15 is regulated by $\mathrm{CHOP}$ of the unfolded protein response and alleviates NAFLD progression in obese mice. Biochem Biophys Res Commun. 2018;498:388-94.

28. Gjymishka A, Su N, Kilberg MS. Transcriptional induction of the human asparagine synthetase gene during the unfolded protein response does not require the ATF6 and IRE1/XBP1 arms of the pathway. Biochem J. 2009;417:695-703.

29. Schulz J, Avci D, Queisser MA, Gutschmidt A, Dreher LS, Fenech EJ, et al. Conserved cytoplasmic domains promote Hrd1 ubiquitin ligase complex formation for ER-associated degradation (ERAD). J Cell Sci. 2017;130:3322-35.

30. Huo L, Chen R, Zhao L, Shi X, Bai R, Long D, et al. Silver nanoparticles activate endoplasmic reticulum stress signaling pathway in cell and mouse models: the role in toxicity evaluation. Biomaterials. 2015;61:307-15.

31. Hsu VW, Shah N, Klausner RD. A brefeldin A-like phenotype is induced by the overexpression of a human ERD-2-like protein, ELP-1. Cell. 1992;69:625-35.

32. Yahiro K, Tsutsuki H, Ogura K, Nagasawa S, Moss J, Noda M. DAP1, a negative regulator of autophagy, controls SubAB-mediated apoptosis and autophagy. Infect Immun. 2014;82:4899-908.

33. Tsutsuki H, Yahiro K, Suzuki K, Suto A, Ogura K, Nagasawa S, et al. Subtilase cytotoxin enhances Escherichia coli survival in macrophages by suppression of nitric oxide production through the inhibition of NF-KB activation. Infect Immun. 2012;80:3939-51.

34. Inohara N, Ding L, Chen S, Nunez G. Harakiri, a novel regulator of cell death, encodes a protein that activates apoptosis and interacts selectively with survivalpromoting proteins Bcl-2 and Bcl-X(L). EMBO J. 1997;16:1686-94.

35. May KL, Paton JC, Paton AW. Escherichia coli subtilase cytotoxin induces apoptosis regulated by host Bcl-2 family proteins Bax/Bak. Infect Immun. 2010;78:4691-6.

36. D'Osualdo A, Anania VG, Yu K, Lill JR, Kaufman RJ, Matsuzawa S, et al. Transcription factor ATF4 induces NLRP1 inflammasome expression during endoplasmic reticulum stress. PLoS ONE. 2015;10:e0130635.

37. Jeong G, Bae H, Jeong D, Ham J, Park S, Kim HW, et al. A Kelch domain-containing KLHDC7B and a long non-coding RNA ST8SIA6-AS1 act oppositely on breast cancer cell proliferation via the interferon signaling pathway. Sci Rep. 2018;8:12922.

38. Desagher S, Osen-Sand A, Nichols A, Eskes R, Montessuit S, Lauper S, et al. Bidinduced conformational change of Bax is responsible for mitochondrial cytochrome c release during apoptosis. J Cell Biol. 1999;144:891-901.

39. Cuddeback SM, Yamaguchi H, Komatsu K, Miyashita T, Yamada M, Wu C, et al. Molecular cloning and characterization of Bif-1. A novel Src homology 3 domain-containing protein that associates with Bax. J Biol Chem 2001;276:20559-65

40. Ohtsuka T, Ryu H, Minamishima YA, Macip S, Sagara J, Nakayama Kl, et al. ASC is a Bax adaptor and regulates the p53-Bax mitochondrial apoptosis pathway. Nat Cell Biol. 2004;6:121-8.

41. Sawada M, Sun W, Hayes P, Leskov K, Boothman DA, Matsuyama S. Ku70 suppresses the apoptotic translocation of Bax to mitochondria. Nat Cell Biol. 2003;5:320-9.

42. Nomura $M$, Shimizu $S$, Sugiyama $T$, Narita $M$, Ito $T$, Matsuda $H$, et al. 14-3-3 Interacts directly with and negatively regulates pro-apoptotic Bax. J Biol Chem. 2003;278:2058-65.

43. Chipuk JE, Kuwana T, Bouchier-Hayes L, Droin NM, Newmeyer DD, Schuler M, et al. Direct activation of Bax by p53 mediates mitochondrial membrane permeabilization and apoptosis. Science. 2004;303:1010-4.

44. Kaya-Aksoy E, Cingoz A, Senbabaoglu F, Seker F, Sur-Erdem I, Kayabolen A, et al. The pro-apoptotic Bcl-2 family member Harakiri (HRK) induces cell death in glioblastoma multiforme. Cell Death Discov. 2019;5:64.

45. Gurzov EN, Ortis F, Cunha DA, Gosset G, Li M, Cardozo AK, et al. Signaling by IL-1 beta + IFN-gamma and ER stress converge on DP5/Hrk activation: a nove mechanism for pancreatic beta-cell apoptosis. Cell Death Differ. 2009;16: 1539-50

46. Ma C, Ying C, Yuan Z, Song B, Li D, Liu Y, et al. dp5/HRK is a c-Jun target gene and required for apoptosis induced by potassium deprivation in cerebellar granule neurons. J Biol Chem. 2007;282:30901-9.
47. Towers E, Gilley J, Randall R, Hughes R, Kristiansen M, Ham J. The proapoptotic dp5 gene is a direct target of the MLK-JNK-c-Jun pathway in sympathetic neurons. Nucleic Acids Res. 2009;37:3044-60.

48. Sanz C, Horita M, Fernandez-Luna JL. Fas signaling and blockade of Bcr-Abl kinase induce apoptotic Hrk protein via DREAM inhibition in human leukemia cells. Haematologica. 2002;87:903-7.

49. Shim J, Lee JY, Jonus HC, Arnold A, Schnepp RW, Janssen KM, et al. YAP-mediated repression of HRK regulates tumor growth, therapy response, and survival under tumor environmental stress in neuroblastoma. Cancer Res. 2020;80:4741-53.

50. Sakuratani T, Takeuchi T, Yasufuku I, Iwata Y, Saigo C, Kito Y, et al. Downregulation of ARID1A in gastric cancer cells: a putative protective molecular mechanism against the Harakiri-mediated apoptosis pathway. Virchows Arch. 2020;478:401-11.

51. Igarashi $T$, Izumi $H$, Uchiumi $T$, Nishio $K$, Arao T, Tanabe $M$, et al. Clock and ATF4 transcription system regulates drug resistance in human cancer cell lines. Oncogene. 2007;26:4749-60.

\section{ACKNOWLEDGEMENTS}

This study was supported by grants-in-aid for Scientific Research, Takeda Science Foundation, and Research Program on Emerging and Re-emerging Infectious Diseases from Japan Agency for Medical Research and Development, AMED (21fk0108611h0701). Joel Moss was supported by the Intramural Research Program, National Institutes of Health, National Heart, Lung, and Blood Institute. We acknowledge the expert technical assistance of K. Hirano at Chiba university.

\section{AUTHOR CONTRIBUTIONS}

$\mathrm{KY}, \mathrm{KO}$ and $\mathrm{HT}$ experimental design, cell signaling, data analysis of RNAseq, writing the paper. SI and MO, data analysis; JM, experimental design, review the paper.

\section{COMPETING INTERESTS}

The authors declare no conflict of interest.

\section{ADDITIONAL INFORMATION}

Supplementary information The online version contains supplementary material available at https://doi.org/10.1038/s41420-021-00753-0.

Correspondence and requests for materials should be addressed to Kinnosuke Yahiro.

Reprints and permission information is available at http://www.nature.com/ reprints

Publisher's note Springer Nature remains neutral with regard to jurisdictional claims in published maps and institutional affiliations.

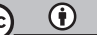

Open Access This article is licensed under a Creative Commons Attribution 4.0 International License, which permits use, sharing, adaptation, distribution and reproduction in any medium or format, as long as you give appropriate credit to the original author(s) and the source, provide a link to the Creative Commons license, and indicate if changes were made. The images or other third party material in this article are included in the article's Creative Commons license, unless indicated otherwise in a credit line to the material. If material is not included in the article's Creative Commons license and your intended use is not permitted by statutory regulation or exceeds the permitted use, you will need to obtain permission directly from the copyright holder. To view a copy of this license, visit http://creativecommons. org/licenses/by/4.0/.

(c) The Author(s) 2021 